\title{
Preoperative toxoplasma gondii serostatus does not affect long-term survival of cardiac transplant recipients: analysis of the Spanish Heart Transplantation Registry
}

\author{
Eduardo Barge-Caballero, ${ }^{\mathrm{a}, \mathrm{q}}$, Luis Almenar-Bonet ${ }^{\mathrm{b}}$, María G. Crespo-Leiro ${ }^{\mathrm{a}, \mathrm{q}}$, \\ Vicens Brossa-Loidi ${ }^{\mathrm{c}}$, Diego Rangel-Sousa ${ }^{\mathrm{d}}$, Manuel Gómez-Bueno ${ }^{\mathrm{e}, \mathrm{q}}$, Marta \\ Farrero-Torres ${ }^{\mathrm{f}}$, Beatriz Díaz-Molina ${ }^{\mathrm{g}}$, Juan Delgado-Jiménez ${ }^{\mathrm{h}, \mathrm{q}}$, Manuel \\ Martínez-Sellés ${ }^{\mathrm{i}, \mathrm{q}}$, Amador López-Granados ${ }^{\mathrm{j}}$, Luis De-la-Fuente-Galán ${ }^{\mathrm{k}}$, José \\ González-Costello $^{1}$, Iris P. Garrido-Bravo ${ }^{\mathrm{m}, \mathrm{q}}$, Teresa Blasco-Peirón ${ }^{\mathrm{n}}$, Gregorio \\ Rábago-Juan-Aracil ${ }^{\circ}$, Francisco González-Vílchez ${ }^{\mathrm{p}}$
}

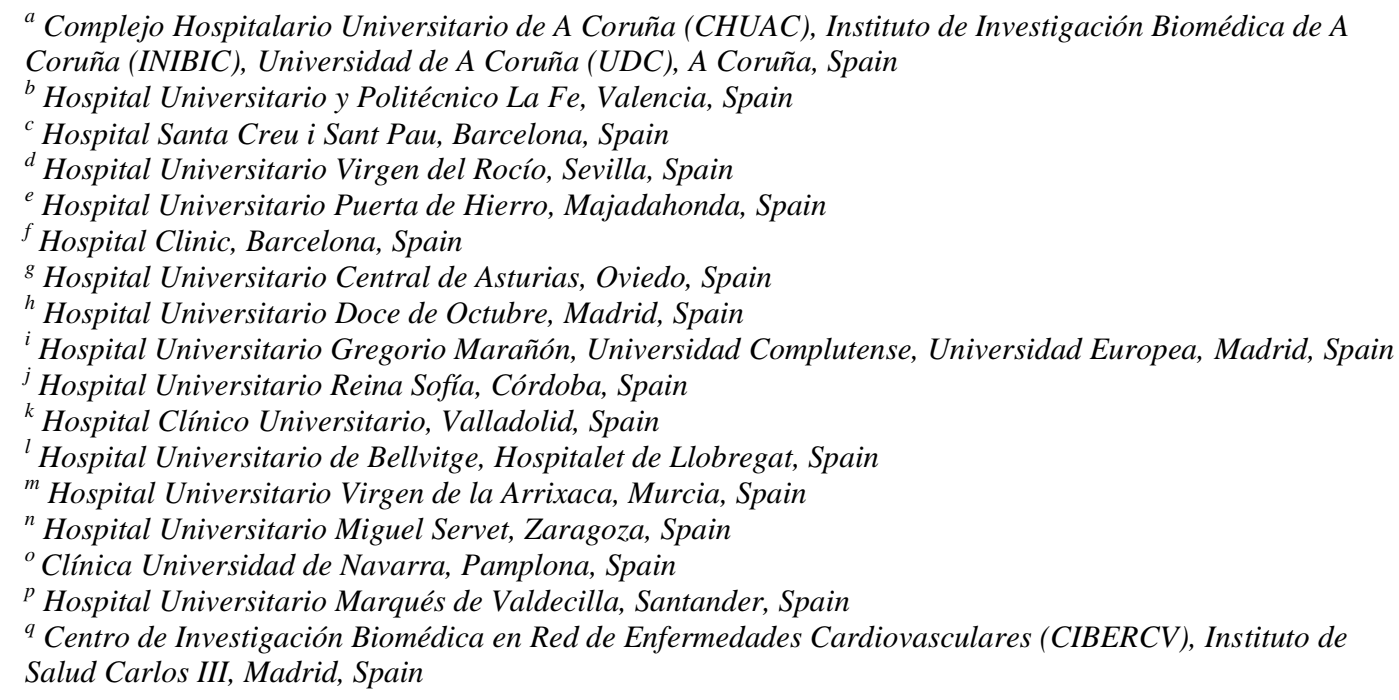

\begin{abstract}
Background. It's unclear whether pre-transplant T. gondii seropositivity is associated with impaired survival in heart transplant recipients.

Objectives. To test the above-mentioned hypothesis in the Spanish Heart Transplantation Registry.

Methods. Post-transplant outcomes of 4048 patients aged > 16 years who underwent first, single-organ heart transplantation in 17 Spanish institutions from 1984 to 2014 were studied. Long-term post-transplant survival and survival free of cardiac death or retransplantation of $2434(60 \%) \mathrm{T}$. gondii seropositive recipients and $1614(40 \%)$ T. gondii seronegative recipients were compared.

Results. T. gondii seropositive recipients were older, had higher body mass index, and presented higher prevalence of hypertension, hypercholesterolemia, COPD and Cytomegalovirus seropositivity than T. gondii seronegative recipients. In univariable analysis, pre-transplant $\mathrm{T}$. gondii seropositivity was associated with increased post-transplant all-cause mortality (non-adjusted HR 1.15; 95\% CI 1.04-1.26). However, this effect was no longer statistically significant after multivariable adjustment by recipient's age and sex (adjusted HR $1.01,95 \%$ CI $0.92-1.11$ ). Extended multivariable adjustment by other potential confounders showed similar results (adjusted HR 0.99, 95\% CI 0.89-1.11). T. gondii seropositivity had no significant effect on the composite outcome cardiac death or retransplantation (non-adjusted HR 1.08, 95\% CI 0.95-1.24, $p=0.235$ ). The distribution of the causes of death was comparable in T. gondii seropositive and T. gondii seronegative recipients. No statistically significant impact of donor's T. gondii serostatus or donor-recipient $\mathrm{T}$. gondii serostatus matching on post-transplant survival was observed.
\end{abstract}


Conclusions. Our analysis did not show a significant independent effect of preoperative T. gondii serostatus on long-term outcomes after heart transplantation.

\author{
Abbreviations \\ CAV, coronary allograft vasculopathy; CI, confidence interval; HR, hazard ratio; HT, heart transplantation; \\ TG, Toxoplasma gondii
}

Keywords

Heart transplantation; Toxoplasma gondii; Outcomes; Survival

\title{
1. Background
}

Over the past 10 years, there has been an unsolved controversy about the potential influence of Toxoplasma gondii (TG) serostatus of heart transplantation (HT) recipients on their long-term outcomes after the operation [1]. A pioneer retrospective report from a Norwegian center [2] showed a striking reduction of late post-transplant survival among TG seropositive recipients as compared with seronegative ones, which was attributed to an increased incidence of advanced coronary allograft vasculopathy (CAV). Enhancement of several immune responses triggered by chronic TG infection was proposed as a hypothetical explanation for these intriguing findings.

The validity of the Norwegian analysis was openly questioned due to inconsistencies in the statistical methodology used to assess confusion bias [3]. Later on, three subsequent single-center studies failed to confirm an independent effect of TG serostatus on post-transplant outcomes [46]; moreover, one study reported increased early post-transplant mortality among TG seronegative recipients [7]. Until now, no significant influence of either donor's TG serostatus or donorrecipient TG serostatus matching on post-transplant outcomes has been proven $[2,4-6]$.

Given the discrepancies among previously published single-center studies, we aimed to investigate a potential impact of recipient's TG serostatus on long-term post-transplant survival in the multi-institutional cohort of the Spanish Heart Transplantation Registry [8].

\section{Methods}

\subsection{Study description}

The Spanish Heart Transplantation Registry is a prospective database promoted by the Working Group in Heart Failure of the Spanish Society of Cardiology that contains detailed clinical information about all HT procedures performed in our nation since 1984 to present. The registry is updated in a yearly basis with data supplied by all transplant centers of the country. This database has been described elsewhere [8].

For the present study, we selected from the Spanish Heart Transplantation Registry database all patients aged > 16 years who underwent first, single-organ, orthotopic HT in Spain until December 31st, 2014, for whom reliable information about their pre-transplant TG serostatus was available. Vital status as of December 31st, 2015 was known for all subjects.

Baseline clinical characteristics and long-term post-transplant survival of TG seropositive and TG seronegative recipients were compared. Pre-transplant TG seropositivity was defined by the detection of specific immunoglobulin $G$ against the parasite, according to local diagnostic protocols of participating hospitals. This investigation was approved by the Local Committee for Ethics in Clinical Research of A Coruña-Ferrol, Department of Healthcare, Autonomous Community of Galicia (Spain). 


\subsection{End-points}

Long-term survival after HT was the primary end-point of the study. The composite outcome cardiac death or cardiac re-transplantation was a secondary end-point.

Cardiac death was defined as any death attributable to heart failure, myocardial ischemia or arrhythmia, including deaths secondary to acute rejection, CAV, or primary or unexplained graft dysfunction. For survival analysis, sudden deaths and deaths from an unknown origin were also assumed as cardiac deaths.

\subsection{Statistical analysis}

In this manuscript, continuous variables are presented as mean \pm standard deviation or median (interquartile rank), depending on whether they follow a normal distribution, and categorical variables are presented as proportions. Baseline clinical characteristics of TG seropositive and TG seronegative recipients were compared by means of the chi-squared test, the T-student test or the Mann-Whitney test, as required.

Non-adjusted long-term post-transplant survival curves of TG seropositive and TG seronegative recipients were constructed with the Kaplan-Meier method and compared with the $\log$ rank test.

Cox's multivariable models were used to control the influence of confusion bias on the statistical effect of recipient's TG serostatus on long-term post-transplant survival. A first multivariable model (Model 1) including recipient's age, recipient's gender and recipient's TG serostatus was constructed in order to adjust the statistical effect of TG seropositivity on survival by demographic factors. Further adjustment was performed by means of an extended multivariable model (Model 2), which included several baseline variables additional to demographic factors, which were considered by the investigators as potential confounders. A confounder was defined as a baseline variable showing an asymmetric distribution between TG seropositive and TG seronegative patients, together with either a statistical significant association with post-transplant survival in the study population or a consistent association with post-transplant survival in previous literature. Covariables selected for extended multivariable adjustment (Model 2) were age of the recipient, gender of the recipient, body mass index, diabetes, hypertension, ischemic heart disease, Cytomegalovirus serostatus, age of the donor, mechanical circulatory support, mechanical ventilation, tacrolimus use, mophetil mycophenolate use, transplant era, and cold ischemic time. Adjusted long-term post-transplant survival curves for TG seropositive and TG seronegative recipients were estimated by means of both multivariable models [1 and 2].

In the subpopulation of subjects for whom both recipient's and donor's TG serostatus were known, we performed an exploratory analysis about a potential influence of donor-recipient TG serostatus matching on long-term post-transplant survival, by using the Kaplan-Meier method and multivariable Cox's regression. For this analysis, patients were divided in four categories according to donor-recipient TG serostatus matching, as follows: donor $(+) /$ recipient $(+)$, donor $(+) /$ recipient $(-)$, donor $(-) /$ recipient $(+)$, and donor $(-)$ /recipient $(-)$. The donor $(+) /$ recipient $(+)$ subgroup was considered as the reference category for statistical comparisons.

Statistical analysis was performed with Stata 12. Statistical significance was set as a $p$ value $<0.05$ for all tests. 


\section{Results}

\subsection{Patients}

From 1984 to 2014, 7277 patients underwent first, single-organ, orthotopic HT in 17 Spanish institutions, 4048 of which constituted the study population. Reasons for exclusion were age $<16$ years $(n=390)$, re-transplantation $(n=164)$, multi-organ transplantation $(n=124)$ and the lack of information about recipient's pre-transplant TG serostatus $(n=2533)$. Before HT, 2434 (60\%) study patients were TG seropositive and 1614 (40\%) were TG seronegative.

\subsection{Baseline clinical characteristics}

The Table 1 shows a comparison of baseline clinical characteristics of TG seropositive and TG seronegative recipients. At the time of HT, TG seropositive recipients were significantly older, had higher body mass index and higher prevalence of cardiovascular risk factors like hypertension and hypercholesterolemia, and more frequently presented chronic obstructive pulmonary disease and Cytomegalovirus seropositivity than TG seronegative recipients.

The need for preoperative mechanical ventilation and mechanical circulatory support was more frequent among TG seronegative recipients; cold ischemic times were also longer in these individuals.

The use of tacrolimus and mycophenolate mophetil as part of the initial immunosuppressive regimen was less frequent among TG seropositive recipients, which in a higher proportion were transplanted during the older era (1984-2000). 
Table 1. Baseline clinical characteristics of $T$. gondii seronegative recipients and $T$. gondii seropositive recipients.

\begin{tabular}{|c|c|c|c|}
\hline & $\begin{array}{l}\text { Toxoplasma gondii seropositive } \\
\text { recipients } \\
N=2434\end{array}$ & $\begin{array}{l}\text { Toxoplasma gondii } \\
\text { seronegative recipients } \\
N=1614\end{array}$ & $\mathrm{p}$ \\
\hline \multicolumn{4}{|l|}{ Recipients } \\
\hline Age (years), mean \pm standard deviation & $54.9 \pm 9.6$ & $48.7 \pm 12.9$ & $<0.001$ \\
\hline Body mass index & $26 \pm 3.9$ & $25 \pm 4.1$ & $<0.001$ \\
\hline Women & $18.1 \%$ & $22.7 \%$ & $<0.001$ \\
\hline Transplant era & & & $<0.001$ \\
\hline 1984-2000 & $30.2 \%$ & $24.2 \%$ & \\
\hline 2001-2014 & $69.8 \%$ & $75.8 \%$ & \\
\hline Ischemic heart disease & $32.9 \%$ & $30.1 \%$ & 0.065 \\
\hline History of smoking & $20.2 \%$ & $19.5 \%$ & 0.638 \\
\hline Diabetes mellitus & $16.2 \%$ & $14.3 \%$ & 0.112 \\
\hline Hypertension & $34.4 \%$ & $28.1 \%$ & $<0.001$ \\
\hline Hypercholesterolemia & $41.2 \%$ & $34.3 \%$ & $<0.001$ \\
\hline Previous cardiac surgery & $23.5 \%$ & $21.6 \%$ & 0.166 \\
\hline Cytomegalovirus seropositive recipient & $86.1 \%$ & $74.2 \%$ & $<0.001$ \\
\hline Previous malignancy & $3.5 \%$ & $4.4 \%$ & 0.153 \\
\hline Chronic obstructive pulmonary disease & $12.5 \%$ & $8.3 \%$ & $<0.001$ \\
\hline Inotropic therapy & $29.6 \%$ & $34.7 \%$ & 0.001 \\
\hline Mechanical circulatory support & $17.6 \%$ & $22.5 \%$ & 0.003 \\
\hline Dialysis & $8.3 \%$ & $9.1 \%$ & 0.435 \\
\hline Mechanical ventilation & $9.5 \%$ & $12.5 \%$ & 0.004 \\
\hline Renal dysfunction $^{\mathrm{a}}$ & $16 \%$ & $17.2 \%$ & 0.329 \\
\hline Liver dysfunction $^{\mathrm{b}}$ & $17.3 \%$ & $16.8 \%$ & 0.690 \\
\hline Mean pulmonary pressure $(\mathrm{mm} \mathrm{Hg})$ & $30.1 \pm 10.6$ & $30.3 \pm 10.6$ & 0.371 \\
\hline Pulmonary vascular resistance (Wood units) & $2.4 \pm 2.2$ & $2.2 \pm 1.3$ & 0.066 \\
\hline \multicolumn{4}{|l|}{ Donors } \\
\hline Cold ischemic time (min) & $184.8 \pm 64.7$ & $191.0 \pm 65.8$ & 0.009 \\
\hline Women & $30.4 \%$ & $33.6 \%$ & 0.034 \\
\hline Body mass index (kg/m2) & $25.2 \pm 3.6$ & $25.1 \pm 3.6$ & 0.289 \\
\hline Age (years) & $35.9 \pm 13.3$ & $35.8 \pm 13.2$ & 0.719 \\
\hline \multicolumn{4}{|l|}{ Baseline immunosuppression } \\
\hline Corticosteroids & $98 \%$ & $97.1 \%$ & 0.506 \\
\hline Cyclosporine A & $63.6 \%$ & $55.6 \%$ & $<0.001$ \\
\hline Tacrolimus & $31.9 \%$ & $39.8 \%$ & $<0.001$ \\
\hline Mycophenolate mophetil & $64.5 \%$ & $70.4 \%$ & $<0.001$ \\
\hline Azathioprine & $32.8 \%$ & $25.5 \%$ & $<0.001$ \\
\hline Sirolimus & $1.4 \%$ & $1.2 \%$ & 0.575 \\
\hline Everolimus & $1.8 \%$ & $2.4 \%$ & 0.224 \\
\hline
\end{tabular}

\footnotetext{
${ }^{\text {a }}$ Serum creatinin $>2 \mathrm{mg} / \mathrm{dl}$.

${ }^{\mathrm{b}}$ Serum bilirrubin $>2 \mathrm{mg} / \mathrm{dl}$.
} 


\subsection{Causes of death}

Median follow-up after HT was 2103 days (interquartile rank 568 to 4153 days). Over this period, 1903 (47\%) patients died, of which 1215 were TG seropositive and 688 were TG seronegative before HT. Also, 79 (2\%) patients underwent cardiac re-transplantation, of which 48 were TG seropositive and 31 were TG seronegative before their first HT.

The presumed cause of death was cardiac in $616(32 \%)$ deceased patients and non-cardiac in $1038(55 \%)$ deceased patients; in the remaining 249 (13\%) deceased patients, it was unknown. The distribution of the causes of death among TG seronegative recipients and TG seropositive recipients showed no relevant differences, as detailed in the Table 2.

Table 2. Distribution of the causes of death of $T$. gondii seropositive recipients and $T$. gondii seronegative recipients.

\begin{tabular}{lll} 
& $\begin{array}{l}T . \text { gondii seronegative } \\
\text { recipients } \\
N=688\end{array}$ & $\begin{array}{l}\text { T. gondii seropositive } \\
\text { recipients } \\
N=1215\end{array}$ \\
& & \\
Cardiac & $233(34 \%)$ & $383(32 \%)$ \\
Graft failure, postoperative & 76 & 119 \\
Graft failure, acute rejection & 29 & 37 \\
Graft failure, coronary allograft vasculopathy & 26 & 62 \\
Graft failure, non-specified & 36 & 58 \\
Sudden death & 66 & 107 \\
Non-cardiac & $364(53 \%)$ & $674(55 \%)$ \\
Multi-organ failure & 109 & 128 \\
Infection & 65 & 162 \\
Malignancy & 47 & 117 \\
Other & 143 & 267 \\
Unkwnown & $91(13 \%)$ & $158(13 \%)$ \\
& & \\
\hline
\end{tabular}

\subsection{Primary end-point: all-cause death}

Kaplan-Meier long-term post-transplant survival curves of TG seropositive and TG seronegative recipients are depicted in Fig. 1. TG seropositive recipients had shorter non-adjusted survival than seronegative ones (non-adjusted hazard ratio (HR) 1.15; 95\% Confidence Interval (CI) 1.04-1.26; log rank $p<0.001)$. Non-adjusted post-transplant all-cause mortality rates were 62.6 (95\% CI 58.1-67.5) deaths per 1000 patients-year among TG seronegative recipients and 70.2 (95\% CI 66.4-74.3) deaths per 1000 patients-year among TG seropositive ones. 


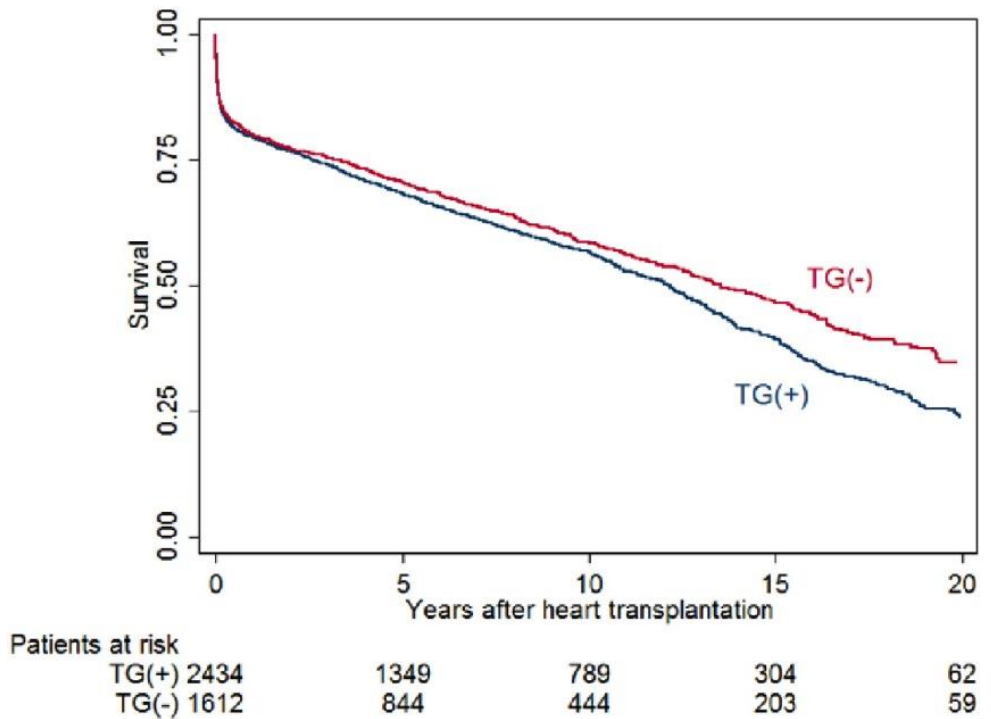

Fig. 1. Kaplan-Meier analysis of long-term post-transplant survival of $T$. gondii seropositive recipients (blue line) and T. gondii seronegative recipients (red line). TG, Toxoplasma gondii.

The statistical effect of TG serostatus on long-term post-transplant survival was no longer statistically significant after multivariable adjustment (Supplementary Table 1). By means of the multivariable model 1, which included the demographic factors age and sex of the recipient as covariables, the estimated HR for death for TG seropositive recipients vs. TG seronegative ones was $1.01(95 \%$ CI $0.92-1.11, p=0.842)$.

Extended multivariable adjustment was conducted by means of multivariable model 2, which included several potential confounders additionally to demographic factors, but no significant change of the results was obtained. By means of the model 2, the estimated HR for death for TG seropositive recipients vs. TG seronegative ones was 0.99 (95\% CI $0.89-1.11, p=0.925)$.

Adjusted long-term post-transplant survival functions of TG seropositive recipients and TG seronegative recipients, as estimated by means of Cox's multivariable models 1 and 2 , are represented graphically in Fig. 2 . 

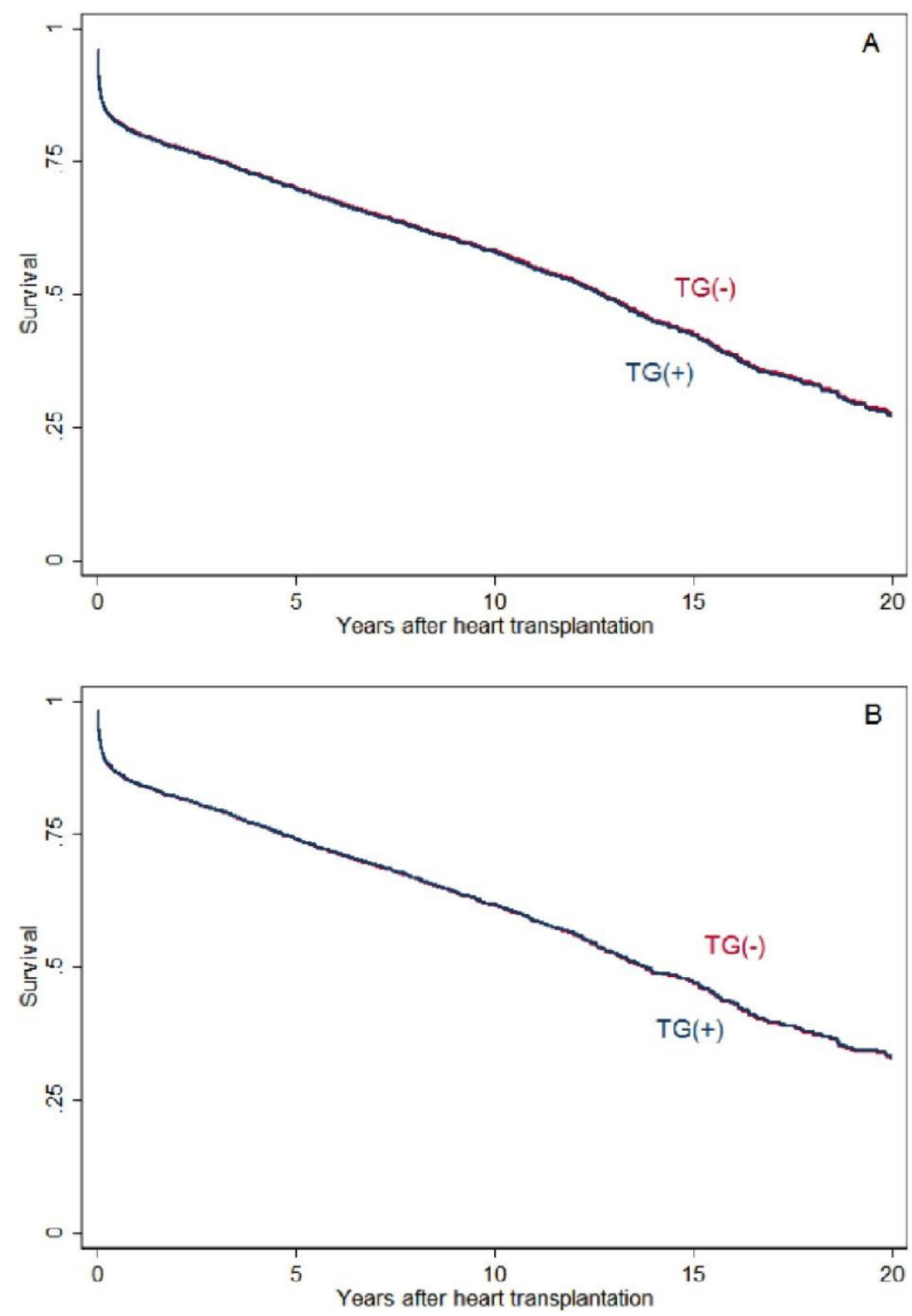

Fig. 2. Adjusted survival functions of $T$. gondii seropositive recipients (blue line) and $T$. gondii seronegative recipients (red line), as estimated by means of Cox's proportional hazards regression. Panel A, Multivariable model 1 (age- and sex-adjusted survival). Panel $\mathrm{B}$, Multivariable model 2 (extended multivariable adjustment).

\subsection{Secondary end-point: cardiac death or cardiac re-transplantation}

Univariable analysis did not reveal a statistically significant association between TG seropositivity and the risk of the composite outcome cardiac death or cardiac re-transplantation (non-adjusted HR 1.08, 95\% CI 0.95-1.24; $p=0.235$ ). Non-adjusted incidence rates of the secondary end-point were 32.1 (95\% CI 29.0-35.7) events per 1000 patients-year among TG seronegative recipients and 34.1 (95\% CI 31.4-36.9) events per 1000 patients-year among TG seronegative ones.

Neither multivariable adjustment by age and sex of the recipient (Model 1: adjusted HR 1.08, 95\% CI $0.95-1.24 ; p=0.231$ ) nor by other potential confounders (Model 2: adjusted HR 1.04, $95 \%$ CI $0.88-1.22 ; p=0.650$ ) led to a significant change of the result. 


\subsection{Donor-recipient T. gondii serostatus matching}

Donor's TG serostatus was known in 1975 (49\%) recipients. Among them, 559 (28\%) were donor $(+)$ /recipient $(+), 302(15 \%)$ were donor $(+) /$ recipient $(-), 620$ (32\%) were donor $(-) /$ recipient $(+)$ and $493(25 \%)$ were donor $(-) /$ recipient $(-)$.

Regardless donor's TG serostatus, TG seronegative recipients showed a trend to higher nonadjusted post-transplant survival than seronegative ones; however, differences across four categories of donor-recipient TG serostatus matching did not reach statistical significance (p log rank $=0.083$, Supplementary Fig. 1).

Considering the donor $(+) /$ recipient $(+)$ subgroup as the reference category, age- and sexadjusted HR (Model 1) for post-transplant all-cause mortality in the donor $(+) /$ recipient $(-)$, donor $(-)$ /recipient $(+)$ and donor $(-) /$ recipient $(-)$ subgroups were $0.92(95 \%$ CI $0.73-1.15 ; p=0.448)$, 1.04 (95\% CI 0.87-1.23; $p=0.676$ ), and 1.02 (95\% CI 0.84-1.24; $p=0.835$ ), respectively. Extended multivariable adjustment (Model 2) showed similar results.

\section{Discussion}

To the best of our knowledge, this is the first multi-institutional study to address the hypothesis that pre-transplant TG seropositivity is a risk factor for mortality in HT recipients. By using a previously collected dataset of $>4000$ patients who received a heart graft in Spain over the last 30 years, we failed to demonstrate an independent impact of pre-transplant TG serostatus on posttransplant survival. Neither a significant effect of donor-recipient TG serostatus matching on posttransplant outcomes was observed.

Since the early eras of transplantation, doctors taking care of transplant recipients are concerned about the possibility of opportunistic infections in such patients. T. gondii is an intracellular parasite, for which the human body constitutes a secondary host. In immune competent subjects, the clinical course of TG infection is usually oligosymptomatic, as the parasite is efficiently cleared by cellular immunity. However, in the setting of cellular immunodeficiency, TG infection may lead to severe clinical consequences, mainly at the central nervous system. Primary infection of a never before exposed subject carries the highest risk of complications; this is the reason why TG serostatus is routinely tested during the selection process of HT candidates, and why primary chemoprophylaxis against TG is usually administered to TG seronegative patients who receive a graft from a TG seropositive donor [9].

Approximately 10 years ago, there was a change in how transplant doctors regard the clinical implications of TG exposure in HT recipients. A striking study based on 288 individuals who underwent HT at a single Norwegian institution reported a strong increase of late post-transplant mortality among TG seropositive recipients in comparison with TG seronegative ones [2]. This finding was attributed to an increased incidence of advanced CAV and death due to this condition. To explain their results, the authors hypothesized that chronic TG infection might contribute to the development of CAV and graft dysfunction through the enhancement of several immunological responses, as endothelial cell activation, cytokine overexpression and T-helper cell activation [2,10-11]. Until now, these pathophysiological mechanisms remain essentially speculative, as no consistent proofs of a causal association between TG exposure and the development of CAV have been presented.

Later on, a few subsequent single-center studies addressed this intriguing topic, but all failed to reproduce earlier findings [4-6]. In their univariable analysis based on 582 patients who underwent cardiac transplantation since 1984 to 2011 in a Dutch center, Van Hellemond et al. [4] also reported reduced non-adjusted long-term post-transplant survival in TG seropositive recipients; however, after multivariable adjustment, the effect of TG serostatus on survival was no longer statistically significant. A Spanish group [5] reached similar conclusions by analyzing the long-term post-transplant outcomes of 657 patients who received a heart graft since 1991 to 2014; in this study, a moderately increased risk of acute rejection was observed among TG seropositive recipients, but this fact did not carry a significant impact on survival. Another single-centre American study focused on 785 patients who underwent HT between 1995 and 2012, showing no 
significant impact of pre-transplant TG serostatus on post-transplant survival [6]. More conflicting results were reported by Doesch et al. [7], who described increased early postoperative mortality among TG seronegative recipients in their analysis based on 344 patients transplanted since 1989 to 2008 in a German institution.

Similarly to described in other studies [4,5], TG seropositive recipients of our multiinstitutional cohort presented shorter non-adjusted long-term post-transplant survival than TG seronegative recipients in univariable analysis. However, this statistically significant, negative effect of pre-transplant TG seropositivity on post-transplant survival was lost after a simple multivariable adjustment by baseline demographic factors -age and sex of the recipient-. A more complex multivariable adjustment, which included several additional covariables selected by study investigators as potential confounders, did not result in a relevant change in the sense of the result.

Our findings suggest that confusion bias is the most probable explanation for the hypothesized association between pre-transplant TG serostatus and post-transplant outcomes. The prevalence of TG exposure, and so, TG seropositivity, is directly related to age [12]. In our series, TG seropositive recipients were, as an average, $>6$ years older than TG seronegative ones at the time of HT. As a result, TG seropositive recipients presented a higher prevalence of adverse clinical conditions related to age, as cardiovascular risk factors, chronic obstructive pulmonary disease and CMV seropositivity. This reality was similar in previous studies $[2,4,5]$.

A glance to the Kaplan-Meier long-term post-transplant survival curves of TG seropositive and TG seronegative recipients only reveals a clear separation between them at late follow-up. In our opinion, this fact reflects the impact of the unbalanced baseline distribution of age and comorbidities in both study groups on long-term post-transplant survival. Interestingly, adjusted HR for all-cause mortality estimated by two different multivariable models were very close to 1 , indicating a consistent neutral effect of TG serostatus on survival. This constitutes a strong argument to affirm that the proposed association between TG serostatus and post-transplant outcomes is probably spurious, rather than causal.

Due to the lack of data, we could not compare the incidence of new-onset CAV or acute rejection in TG seropositive and TG seronegative recipients. Nevertheless, neither univariable nor multivariable analyses revealed a statistically significant difference between both groups with regard to the risk of the composite end-point cardiac death or cardiac re-transplantation, which is an appropriate surrogate for those graft-related adverse clinical events. To date, only one study described an excess of CAV-related mortality in TG seropositive recipients [2]; other authors [4$5,7]$, like us, did not observe any relevant difference with regard to the distribution of the causes of death according to pre-transplant TG serostatus.

Our analysis did not show a significant impact of donor's TG serostatus or donor-recipient TG serostatus matching on post-transplant outcomes. This conclusion may be regarded with caution, given that recipient's TG serostatus was only known in $49 \%$ patients of our population. Nonetheless, this finding is also consistent with previous studies [2,4-7]. The lack of a negative prognostic effect of a TG seropositive donor in a TG seronegative recipient has been proposed as an argument against a causal association between TG serostatus and post-cardiac transplant outcomes [1].

Our study has a few limitations. As a retrospective one, it is exposed to potential selection and information biases inherent to this type of investigation. Comprehensive multivariable adjustment was conducted in order to control the effect of potential confounders; however, we cannot rule out categorically that any other non-tested variable may have influenced observed statistical associations. The study addressed a heterogeneous population, as it involved several hospitals across Spain, with different center-specific protocols regarding post-transplant chemoprophylaxis and infection surveillance. It is remarkable that our analysis excluded near $45 \%$ HT recipients enrolled in the Spanish Heart Transplant Registry; the lack of reliable information about their preoperative TG serology was the reason for exclusion in most cases. The lack of information about evolutionary TG serology prevented us to analyze the incidence of TG new infection or reactivation over post-transplant follow-up. Specific information about the type, duration and compliance of TG chemoprophylaxis was not available. Given the long period studied, a significant era effect cannot be excluded; notwithstanding this, no relevant changes in the 
association between TG serostatus and post-transplant outcomes was observed when transplant era was entered as a covariable in multivariable models constructed for statistical adjustment.

\section{Conclusions}

In conclusion, our study showed a statistically significant, moderate reduction of non-adjusted long-term survival after orthotopic HT among recipients who showed a positive serostatus against TG before the intervention, as compared with seronegative ones. However, the effect of preoperative TG seropositivity on post-transplant survival remained neutral after multivariable adjustment, both in a simple model which only included recipient's age and sex as co-variables, and also in an extended model which added several other potential confounders. Our data suggest that the hypothesized association between recipient's TG serostatus and long-term post-transplant survival is, rather than causal, more probably explained by confusion bias derived by an older age at the time of HT and a higher prevalence of comorbidities among seropositive recipients. Therefore, this study does not support any specific change in the standard clinical management of TG seropositive individuals undergoing orthotopic HT.

\section{Acknowledgements}

A list of study collaborators is provided as Supplementary Material.

\section{Funding}

This study was supported by the Working Group in Heart Failure of the Spanish Society of Cardiology. It was co-financed with European Union Regional Development Funds (EURDF), supplied through the Centro de Investigación Biomédica en Red de Enfermedades Cardiovasculares (CIBERCV), Instituto de Salud Carlos III, Spanish Ministry of Economy and Competitiveness.

\section{Disclosures}

None.

\section{References}

[1] M.M. Kittleson, J. Kobashigawa. Toxoplasma gondii exposure in the heart transplant recipient: good, bad or indiferent?. Transplantation, 96 (2013), p. 1025

[2] S. Arora, P.A. Jenum, P. Aukrust, H. Rollag, A.K. Andreassen, S. Simonsen, et al. Pre-transplant toxoplasma gondii seropositivity among heart transplant recipients is associated with an increased risk of all-cause and cardiac mortality. J. Am. Coll. Cardiol., 50 (2007), pp. 1967-1972

[3] X. Flores-Ríos, M.J. Paniagua-Martín, J. Muñiz-García, M.G. Crespo-Leiro. Pretransplant Toxoplasma gondii seropositivity among heart transplant recipients and mortality. J. Am. Coll. Cardiol., 51 (1827) (2008)

[4] J.J. Van Hellemond, R.T. van Domburg, K. Caliskan, O. Birim, A.H. Balk. Toxoplasma gondii serostatus is not associated with impaired long-term survival after heart transplantation. Transplantation, 96 (2013), pp. 1052-1058

[5] E. Barge-Caballero, C. Barbeito-Caamaño, G. Barge-Caballero, D. Couto-Mallón, M.J. Paniagua-Martín, R. Marzoa-Rivas, et al. Toxoplasma Gondii Serostatus in heart transplant recipients: is it an independent prognostic factor?. Rev. Esp. Cardiol. (Engl. Ed.), 69 (2016), pp. 1160-1166

[6] Chehrazi-Raffle, M. Luu, Z. Yu, F. Liou, M. Kittleson, M. Hamilton, J. Kobashigawa. Toxoplasma gondii serology and outcomes after heart transplantation: contention in the literature. Transplant. Proc., 47 (2015), pp. 1949-1953 
[7] A.O. Doesch, K. Ammon, M. Konstandin, S. Celik, A. Kristen, L. Frankenstein, et al. Negative pretransplant serostatus for Toxoplasma gondii is associated with impaired survival after heart transplantation. Transpl. Int., 23 (2010), pp. 382-389

[8] F. González-Vílchez, J. Segovia Cubero, L. Almenar, M.G. Crespo-Leiro, J.M. Arizón, I. Sousa, et al. Spanish Heart Transplantation Registry. 27th official report of the Spanish Society of Cardiology Working Group on Heart Failure and Heart Transplantation (1984-2015). Rev. Esp. Cardiol. (Engl. Ed.), 69 (2016), pp. 10711082

[9] F. Derouin, H. Pelloux. Prevention of toxoplasmosis in transplant patients. Clin. Microbiol. Infect., 14 (2008), pp. 1089-1101

[10] M. Veldhoen, R.J. Hocking, C.J. Atkins, R.M. Locksley, B. Stockinger. TGFbeta in the context of an inflammatory cytokine milieu supports de novo differentiation of IL17-producing T cells. Immunity, 24 (2006), pp. 179-189

[11] J.P. Woodman, I.H. Dimier, D.T. Bout. Human endothelial cells are activated by IFNgamma to inhibit toxoplasma gondii replication. Inhibition is due to a different mechanism from that existing in mouse macrophages and human fibroblasts. J. Immunol., 147 (1991), pp. 2019-2023

[12] M.J. Gargaté, I. Ferreira, A. Vilares, S. Martins, C. Cardoso, S. Silva, B. Nunes, J.P. Gomes. Toxoplasma gondii seroprevalence in the Portuguese population: comparison of three cross-sectional studies spanning three decades. BMJ Open, 6 (2016), Article $\mathrm{e} 011648$ 